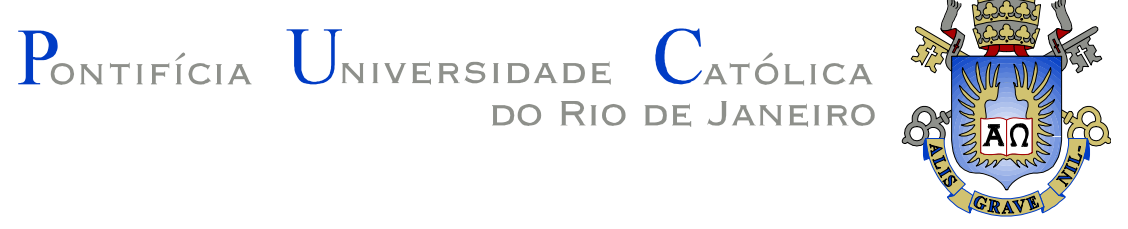

Franky Eduardo Reyes Cac

Análise metrológica do comportamento transiente de um psicrômetro do tipo bulbo úmido e bulbo seco

Dissertação de Mestrado

Dissertação apresentada como requisito parcial para obtenção do título de Mestre pelo Programa de PósGraduação em Metrologia para Qualidade e Inovação da PUC-Rio.

Orientador: Prof. Alcir de Faro Orlando, PhD.

Rio de Janeiro, janeiro de 2005 


\title{
Análise metrológica do comportamento transiente de um psicrômetro do tipo bulbo úmido e bulbo seco
}

\begin{abstract}
Dissertação apresentada como requisito parcial para obtenção do grau de Mestre pelo Programa de Pós-Graduação em Metrologia do Centro Técnico Científico da PUC-Rio. Aprovada pela Comissão Examinadora e homologada pela Coordenação Setorial de Pós Graduação, formalizado pelas respectivas assinaturas.
\end{abstract}

\section{Alcir de Faro Orlando, PhD. \\ Orientador \\ Departamento de Engenharia Mecânica Pontifícia Universidade Católica do Rio de Janeiro (PUC-Rio)}

Nísio de Carvalho Lobo Brum, Dr. URFJ - Universidade Federal do Rio de Janeiro

Paula Medeiros Proença de Gouvêa, PhD.

Programa de Pós-Graduação em Metrologia Pontifícia Universidade Católica do Rio de Janeiro (PUC-Rio)

Marcos Sebastião de Paula Gomes, PhD. Departamento de Engenharia Mecânica Pontifícia Universidade Católica do Rio de Janeiro (PUC-Rio)

Prof. José Eugenio Leal Coordenador Setorial do Centro Técnico Científico (PUC-Rio) 
Todos os direitos reservados. É proibida a reprodução total ou parcial do trabalho sem autorização da universidade, da autora e do orientador.

\section{Franky Eduardo Reyes Cac}

Graduou-se de Físico Aplicado na Faculdade de Engenharia da Universidade de San Carlos de Guatemala em 1994. Cursou o Mestrado em Radioproteção e Dosimetria na área de Física Médica, no Instituto de Radioproteção e Dosimetria IRD/CNEN (RJ-2004).

Atualmente é o responsável do departamento de Física Médica do Instituto de Cancerologia (INCAN) da Guatemala, da transferência e implementação de novos procedimentos de dosimetria física e clínica para o tratamento de câncer, da dosimetria em vivo através da utilização de diodos e da implementação de normas ISO na clínica de radioterapia La Asunción, de Guatemala C.A.

Ficha Catalográfica

Reyes Cac, Franky Eduardo

Análise metrológica do comportamento transiente de um psicrômetro do tipo bulbo úmido e bulbo seco / Franky Eduardo Reyes Cac ; orientador: Prof. Alcir de Faro Orlando. - Rio de Janeiro : PUC-Rio, Programa de Pós-Graduação em Metrologia para Qualidade e Inovação, 2005.

$92 \mathrm{f.} ; 30 \mathrm{~cm}$

Dissertação (mestrado) - Pontifícia Universidade Católica do Rio de Janeiro, Programa de Pós-Graduação em Metrologia para Qualidade e Inovação .

Inclui bibliografia

1. Metrologia - Teses. 2. Psicrômetro. 3. Bulbo úmido. 4. Akaike. 5. Incerteza de medição. 6. Transiente. I. Orlando, Alcir de Faro. II. Pontifícia Universidade Católica do Rio de Janeiro. Programa de Pós-Graduação em Metrologia para Qualidade e Inovação . III. Título. 


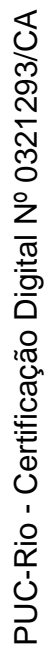

Deus, Criador e Principio de todas as coisas. 


\section{Agradecimentos}

Ao Professor Dr. Maurício Nogueira Frota, Diretor do MQI, pela grande oportunidade de me matricular no mestrado em metrologia, e poder somar esforços no desenvolvimento de meu país.

Ao Professor Dr. Alcir de Faro Orlando, por me orientar na dissertação, pela imensa paciência e consideração durante o desenvolvimento do trabalho, e compartilhar sua experiência na área da metrologia.

À PUC-Rio, pelos auxílios concedidos, sem os quais este trabalho não teria sido realizado.

À Professora Dra. Elisabeth Costa Monteiro pela motivação a me formar em físico biomédico, visando a melhora dos povos mais carentes.

À Dra. Claudia L.P. Maurício, pelo constante apoio para finalizar com sucesso minha formação de metrólogo, cumprindo simultaneamente com minhas tarefas no IRD.

À Sra. Eliane Albernaz e Srta. Marcia Ribeiro, secretárias do Pós MQI/PUC-RJ por garantir que todas as atividades no mestrado foram bem sucedidas.

À Evêmero Calegario e João Peixe, pela grande colaboração técnica durante o desenvolvimento da parte experimental do presente trabalho.

A Jorge Calderón, Mónica Ari, Daniel Louzada e Myrna de Oliveira por sua constante colaboração e valiosos aportes durante todo o mestrado.

Aos Professores da Banca Examinadora. 


\section{Resumo}

Reyes Cac, Franky Eduardo. Análise metrológica do comportamento transiente de um psicrômetro do tipo bulbo úmido e bulbo seco. Rio de Janeiro, 2005. 92p. Dissertação de Mestrado - Departamento de Metrologia para Qualidade e Inovação, Pontifícia Universidade Católica do Rio de Janeiro.

Este trabalho apresenta um estudo do comportamento transiente de um medidor de umidade tipo bulbo úmido e bulbo seco, assim como a determinação da umidade do ar em diferentes condições ambientais, que foram simuladas em laboratório. $\mathrm{O}$ psicrômetro do tipo bulbo úmido e bulbo seco é amplamente utilizado na indústria de ar condicionado, na monitoração da umidade em ambientes fechados, nas estações de meteorologia para a monitoração da umidade da atmosfera, e na prevenção das condições climáticas. Para o desenvolvimento do presente trabalho, foi construído um psicrômetro do tipo bulbo úmido e bulbo seco de ar aspirado, utilizando dois termômetros separados por uma placa de acrílico. Os termômetros utilizados foram tipo PT-100 e tipo Termopar. Na avaliação do conteúdo de umidade do ar, foi utilizada a medição de temperatura registrada por dois termômetros que compõem o psicrômetro, onde um deles registra a temperatura do ar que incide sobre o psicrômetro e serve como referência; e o segundo termômetro, recoberto com uma mecha molhada com água destilada, registra uma temperatura mais baixa devido ao fenômeno de resfriamento evaporativo, o qual é conseqüência da transferência de calor e massa entre a mecha molhada e o ar forçado a passar através dela. Na avaliação do comportamento transiente do psicrômetro, foram aplicados diferentes modelos de ajuste aos dados experimentais, procurando aquele que melhor representasse o comportamento através do tempo do termômetro bulbo úmido. Entre os modelos de ajuste estão: o linear, o exponencial, a soma de dois exponenciais, o racional, o polinomial de grau 6 e o exponencial linear (soma de exponencial com linha reta). A cada um dos modelos foi aplicado o método dos mínimos quadrados, e foi determinado por meio do critério de Akaike que o modelo exponencial linear é o melhor modelo de ajuste. As incertezas das constantes dos diferentes modelos de ajuste foram calculadas através do método das diferenças finitas. São apresentados os valores das constantes de tempo para diferentes valores de velocidade do ar e diferentes graus de umidade, tanto para os termômetros PT-100 quanto para o tipo Termopar. Finalmente, são apresentados os resultados da aplicação da regressão linear múltipla entre as constantes de tempo, a velocidade e a umidade do ar, tanto para o psicrômetro baseado nos termômetros tipo PT-100 quanto nos termômetros tipo Termopar. A presente pesquisa de mestrado contribui para o desenvolvimento da metrologia térmica no Brasil.

\section{Palavras-chave}

Psicrômetro; bulbo úmido; transiente; Akaike; incerteza de medição; metrologia. 


\section{Abstract}

Reyes Cac, Franky Eduardo. Metrological analysis of the transient behavior of a wet bulb and dry bulb psychrometer. Rio de Janeiro, 2005. 92p. MSc. Dissertation Departamento de Metrologia para Qualidade Industrial, Pontifícia Universidade Católica do Rio de Janeiro.

This work presents a study of the transient behavior of a wet bulb and dry bulb psychrometer, as well as the determination of the humidity of air under different environment conditions. The wet bulb and dry bulb psychrometer are widely used in the air conditioning industry, for monitoring the humidity in closed environments, and in meteorology stations for the monitoring the atmosphere humidity and in the prevention of the climatic conditions. For the development of the present work, a wet bulb and dry bulb aspirated psychrometer was built, using two thermometers, which are physically separated by an acrylic plate. Two types of thermometers were used: PT-100 and Thermocouple. In the evaluation of the humidity content, the measurement of temperature was been registered by two thermometers (used as the psychrometer components), where one of them registers the temperature of the air that impacts the psychrometer and serves as reference; and the other one covered by a wick wet with distilled water, registers a lower temperature due to the phenomenon of evaporative cooling, which is a consequence of the mass transfer between the wet wick and the air that is forced to pass through it. In the evaluation of the transient behavior of the psychrometer different models of adjustment to the experimental data have been applied, seeking the best theoretical model that represented the behaviour through the time of the humid bulb thermometer. Among the adjustment models are: the linear one, the exponential one, the addition of two exponential ones, the rational one, polynomial of exponential degree 6 and the linear one (which adds the exponential curve and the straight line). For each of the models the method of the minimum square had been applied, and it was found by means of the of Aikaike's criterion that the linear exponential model is the best one. The uncertainties of the constants of the different models of adjustment were calculated through the method of the finite differences. The values of the time constants of time for different values of air speed and different degrees of humidity for both thermometers PT-100 and Thermocouple type had been presented. Finally, the results of the application of the multiple linear regression are presented among the time constants, the speed, and the humidity of air for both psychrometer based on the thermometers PT-100 type and the thermometers Thermocouple type. This MSc research contributes towards the development of the thermal metrology in Brazil.

\section{Keywords}

Psychrometer; wet bulb; transient; Akaike; uncertainty in measurement; metrology. 


\section{Sumário}

1 Introdução 17

1.1. Justificativa do presente trabalho 17

1.2. Objetivo 19

$\begin{array}{ll}\text { 1.3. Metas } & 19\end{array}$

2 Considerações Teóricas 20

2.1. Definição de gás 20

2.2. Gás perfeito 20

2.2.1. Considerações sobre misturas de gases perfeitos 20

2.3. Umidade 21

2.3.1. Definições e parâmetros de umidade 22

2.4. Métodos para medição da umidade 24

2.4.1. Padrões de umidade 24

2.4.2. Métodos de medição de umidade em gases industriais 28

2.5. Psicrometria 30

2.6. O higrômetro bulbo úmido e bulbo seco 37

2.6.1. Teoria do termômetro tipo bulbo úmido 38

3 Materiais, Procedimentos Experimentais e Análise de Dados. 44

3.1. Materiais e equipamento 44

3.1.1. Termômetros PT-100 44

3.1.2. Termômetros termopar tipo T 46

3.1.3. Higrômetro tipo bulbo úmido e bulbo seco 47

3.1.4. Programa de aquisição de dados LabView 48

3.1.5. Sistema Pneumático de ar comprimido 49

3.1.6. Sistema de umidificação do ar 51

3.1.7. Aquisição digital de dados 51

3.2. Procedimentos experimentais 51

3.2.1. Determinação da umidade relativa 51

3.2.2. Comportamento transiente do psicrômetro 52 
3.3. Construção dos modelos matemáticos para os dados experimentais.

3.3.1. Ferramentas para a construção de um modelo matemático

3.3.2. Método dos mínimos quadrados linear aplicados ao ajuste de dados experimentais

3.3.3. Método dos mínimos quadrados não linear aplicados ao ajuste de dados experimentais

3.4. Comparação e escolha do melhor modelo de ajuste de dados experimentais

3.4.1. Base estatística para a comparação de modelos de ajuste

3.4.2. Critério da Informação de Akaike (AIC)

4 Resultados e Analise

4.1. Avaliação preliminar 66

4.2. Determinação da umidade relativa utilizando os termômetros PT-100 68

4.3. Determinação da umidade relativa utilizando termopares

4.4. Determinação da constante de tempo através de $\ln [(\mathrm{T}-\mathrm{Tf}) /(\mathrm{Ti}-\mathrm{Tf})]$ para o psicrômetro utilizando termômetros PT-100.

4.4.1. Determinação das constantes de tempo segundo o modelo logarítmico 71

4.5. Determinação da constante de tempo através de $\ln [(\mathrm{T}-\mathrm{Tf}) /(\mathrm{Ti}-\mathrm{Tf})]$ para o psicrômetro utilizando termopares

4.5.1. Determinação das constantes de tempo segundo o modelo logarítmico 73

4.6. Comportamento transiente do psicrômetro tipo bulbo úmido e bulbo seco baseado em termômetros tipo PT-100 e Termopar

4.7. Resultados do comportamento transiente do psicrômetro utilizando termômetros PT-100 e termômetros Termopar.

4.8. Constantes do modelo exponencial + linear que melhor ajusta os dados experimentais através do tempo do psicrômetro tipo bulbo úmido e bulbo seco baseado na utilização de termômetros tipo PT-100.

4.9. Constantes do modelo exponencial + linear que melhor ajusta os dados experimentais através do tempo do psicrômetro tipo bulbo úmido e bulbo seco baseado na utilização de termômetros tipo Termopar.

4.10. Relação linear entre as quantidades de interesse na utilização do 
psicrômetro de bulbo úmido e a constante de tempo determinada pelo modelo exponencial linear.

5 Conclusões

6 Referências Bibliográficas

Anexo A 


\section{Lista de símbolos}

A Constante psicrométrica

$\mathrm{A}^{-1} \quad$ Matriz inversa de $\mathrm{A}$

AIC Critério de Akaike

$\mathrm{C}_{\mathrm{pa}} \quad$ Calor especifico do ar seco a pressão constante

$\mathrm{C}_{\mathrm{pm}} \quad$ Calor especifico de uma massa de ar a pressão constante

$\mathrm{C}_{\mathrm{pv}} \quad$ Calor especifico do vapor de água a pressão constante

$\mathrm{C}_{\mathrm{vm}} \quad$ Calor especifico do ar úmido a volume constante

$\mathrm{D}^{\mathrm{T}} \quad$ Matriz transposta de D

e Pressão de vapor real

$\mathrm{e}_{\mathrm{s}} \quad$ Pressão de vapor de saturação

$e_{s, b s} \quad$ Pressão de saturação na temperatura do bulbo seco

$\mathrm{e}_{\mathrm{s}, \mathrm{bu}} \quad$ Pressão de saturação na temperatura do bulbo úmido

h Entalpia especifica

$\boldsymbol{h}$ Coeficiente de transferência de calor

L Entalpia de evaporação

$\mathrm{M}_{\mathrm{ar}} \quad$ Massa molecular média do ar atmosférico

P Pressão

ph Saturação percentual

Q Vazão da turbina

$\mathrm{R}_{\mathrm{m}} \quad$ Constante de gás equivalente para a mistura ar-vapor

$\mathrm{R}_{\mathrm{V}} \quad$ Constante do gás para o vapor

SSR Soma quadrática de resíduos

T Temperatura em graus Celsius

$\mathrm{T}_{\mathrm{bs}} \quad$ Temperatura do bulbo seco

$\mathrm{T}_{\mathrm{bu}} \quad$ Temperatura do bulbo úmido

$\mathrm{V} \quad$ Volume do gás

V Matriz de covariância

$\mathrm{V}_{\mathrm{m}} \quad$ Volume especifico real do ar úmido não saturado 
$\mathrm{X}_{\mathrm{s}, \mathrm{bs}} \quad$ Conteúdo de umidade na temperatura do bulbo seco

W* Razão de umidade

\section{Lista de símbolos gregos}

$\phi \quad$ Umidade relativa

$\rho_{\mathrm{m}} \quad$ Massa especifica real do ar úmido

$\sigma^{2} \quad$ Variância

$\tau \quad$ Constante de tempo

$\chi_{v} \quad$ Fração molar de vapor

$\chi_{v s} \quad$ Fração molar de vapor saturado 


\section{Lista de figuras}

Figura 1. Hierarquia dos padrões de umidade no NBS (Hasegawa, 1986). 25

Figura 2. Balanço de calor no bulbo úmido. 39

Figura 3. Desenho esquemático para a construção do Psicrômetro. 48

Figura 4. Tela principal do programa de aquisição de dados em LabView®. 49

Figura 5. Velocidade do ar $x$ freqüência da turbina 50

Figura 6. Multímetro digital Agilent 34401A. 51

Figura 7. Caixa de dialogo de Solver de Excel 58

Figura 8. Curva de temperatura versus tempo para uma velocidade do ar de $1,44 \mathrm{~m} / \mathrm{s}$. 66

Figura 9. Psicrômetro tipo bulbo úmido e bulbo seco construído para o presente trabalho. $\quad 67$

Figura 10. Posição dos termômetros tipo bulbo úmido e bulbo seco PT-100 e termopar. $\quad 67$

Figura 11. Arranjo experimental utilizado no presente trabalho. 67

Figura 12. Gráfico $y=\ln \left[\left(\left(T-T_{f}\right) /\left(T_{i}-T_{f}\right)\right]\right.$ contra o tempo, com o PT-100 70

Figura 13. Curvas de $y=\ln \left[\left(\left(T-T_{f}\right) /\left(T_{i}-T_{f}\right)\right]\right.$ contra o tempo, com cortes inicial e final para o PT-100. 71

Figura 14. Gráfico $y=\ln \left[\left(\left(T-T_{f}\right) /\left(T_{i}-T_{f}\right)\right]\right.$ contra o tempo, com os termopares.

Figura 15. Curvas de $y=\ln \left[\left(\left(T-T_{f}\right) /\left(T_{i}-T_{f}\right)\right]\right.$ contra o tempo, com cortes inicial e final para o Termopar.

Figura 16. Comportamento transiente do psicrômetro utilizando termômetros PT-100.

Figura 17. Comportamento transiente do psicrômetro utilizando termômetros Termopar.

Figura 18. Curvas experimentais do comportamento transiente do bulbo úmido para diferentes velocidades do ar e diferentes graus de umidade com PT100. 
Figura 19. Curvas experimentais do comportamento transiente do bulbo úmido para diferentes velocidades do ar e diferentes graus de umidade com Termopar. $\quad 76$

Figura 20. Curvas experimentais do bulbo úmido utilizando termômetros PT100.

Figura 21. Curvas experimentais do bulbo úmido utilizando termômetros Termopar. 


\section{Lista de tabelas}

Tabela 1. Alguns sais existentes no mercado e suas respectivas temperaturas de equilíbrio. 29

Tabela 2. Componentes do ar atmosférico. 31

Tabela 3. Dados da calibração do PT-100-03 45

Tabela 4. Dados da calibração do PT-100-06 46

Tabela 5. Dados da calibração do Termopar 01

Tabela 6. Dados da calibração do Termopar $02 \quad 47$

Tabela 7. Dados de calibração da turbina. 50

Tabela 8. Velocidade do ar em função da freqüência. 53

Tabela 9. Valor do fator de abrangência $k_{p}$ em função do nível de confiança $p$ para uma distribuição normal. $\quad 61$

Tabela 10. Planilha padrão construída no Excel $₫$ para o calculo da umidade relativa baseada nas medições realizadas com os termômetros PT-100 68

Tabela 11. Valores para diferentes graus de umidade relativa e velocidade do ar utilizando termômetros PT-100. 68

Tabela 12. Valores para diferentes graus de umidade relativa e velocidade do ar utilizando o psicrômetro com termômetros tipo Termopar.

Tabela 13. Constante de tempo do psicrômetro utilizando PT-100 em função velocidade e umidade do ar segundo o modelo logarítmico, as incertezas estão calculadas para $\mathrm{k}=2$.

Tabela 14. Constante de tempo do psicrômetro utilizando Termopar em função velocidade e umidade do ar segundo o modelo logarítmico, as incertezas estão calculadas para $\mathrm{k}=2$.

Tabela 15. Resumo dos valores obtidos na aplicação do critério de AKAIKE para medições feitas com PT-100 na escolha do melhor modelo de ajuste.

Tabela 16. Probabilidade de fazer a escolha do modelo certo utilizando termômetro PT-100, tomando como referência o modelo $\ln [(\mathrm{T}-\mathrm{Te}) /(\mathrm{To}-$ Te)] 
Tabela 17. Resumo dos valores obtidos na aplicação do critério de AKAIKE para medições feitas com Termopar na escolha do melhor modelo de ajuste.

Tabela 18. Probabilidade de fazer a escolha do modelo certo utilizando termômetro Termopar, tomando como referência o modelo $\ln [(\mathrm{T}-\mathrm{Te}) /(\mathrm{To}-$ Te)]

Tabela 19. Valores das constantes do modelo de ajuste para a temperatura do termômetro bulbo úmido PT-100 e valores das condições do ar.

Tabela 20. Valores das constantes do modelo de ajuste para a temperatura do termômetro bulbo úmido Termopar e valores das condições do ar. $\quad 80$

Tabela 21. Resumo de constantes calculadas para medições feitas com PT100

Tabela 22. Resumo de constantes calculadas para medições feitas com Termopares. 